\title{
Guía Documental de Psicología Industrial y Organizacional
}

\section{Fernández Rios; L. Giménez Garcia y J. M. Fernández Dols **}

\section{INTRODUCCION}

El presente artículo trata de ofrecer un panorama bibliográfico de la Psicología Organizacional, especialmente en su dimensión más clásica: la Psicología Industrial.

El estudio de las organizaciones reúne en las Ciencias Sociales nombres muy importantes: Max Weber, Amitai Etzioni, Herbert A. Simon, Elton Mayo, J. K. Galbraith, etc. (vid. por ej., Pugh et al. 1971).

Por otra parte, y como indica Dunnette (1976), la Psicología Organizacional actual ha dejado de ser un conjunto de recetas prácticas sin pretensiones para convertirse en un amplio y complejo panorama teórico en el que se entrelazan puntos de vista provenientes de la Psicologia, la Economia y la Sociología. Se trata, pues, de un marco de referencia que no sólo es útil para el profesional de la
Psicología Aplicada sino también para el investigador del comportamiento humano.

La Guía Documental de Psicología Industrial y Organizacional posee la misma filosofia, ventajas y limitaciones que anteriores Guías sobre Psicología Ambiental (29), Stress (30), Biofeedback (3 I), Ordenadores en Psicologia (32). Una vez más es preciso recordar al lector que se han tenido en cuenta los criterios de practicidad y en ningún caso los de exhaustividad. Las Guias no son un trabajo bibliométrico y, por tanto, los autores se reservan cierto grado de arbitrariedad en función de sus gustos, formación y criterios.

\section{ORGANIZACION DE LA PRESENTE GUIA}

Rompiendo la estructura habitual de las Guías, vamos a distinguir en ésta tres

* Manuscrito elaborado en septiembre de 1983.

** Dirección de los autores: Dpto. de Ecopsicología y Personalidad, Facultad de Psicología, Universidad Autónoma. Campus de Cantoblanco. 28049 Madrid. 
niveles que no se ajustan a la diferenciación en términos documentales entre fuentes primarias y fuentes secundarias.

Dichos tres niveles se acomodan a lo que, creemos, son las tres categorías más frecuentes de usuarios en Psicología de las Organizaciones.

Un primer nivel agrupa las publicaciones en castellano; el volumen de información es en este caso considerablemente superior al de áreas tratadas en anteriores Guías. La selección ha sido inevitable pero los criterios han sido amplios y se ha procurado acompañar las referencias con una breve reseña.

Este primer nivel será especialmente útil para los principiantes en este complejo campo.

El segundo nivel trata de recoger obras solventes de la Psicología Organizacional que no han sido traducidas. Algunas de sus referencias, por ej. el Handbook de Dunnette, deben incluirse también entre las consultas obligadas del primer nivel.

Los criterios de inclusión de estas obras hacen que, en general, se trate de ediciones recientes y que su número sea reducido. Si existe una reseña crítica de la obra ésta se cita en nota a pie de página.

Evidentemente, este segundo nivel no es exhaustivo ni pretende serlo. Puede ser un complemento interesante para el lector del primer nivel $e$ incluso un suplemento de muchas de las obras de aquél. El profesional puede, simplemente, encontrar alguna referencia que le había pasado inadvertida.

El tercer nivel de esta Guía es el más heterogéneo. Incluye las fuentes primarias por excelencia para el profesional especializado, que son las revistas. Para llegar a ellas el lector debe seguir el itinerario que marcan dos fuentes secundarias muy relevantes: las revisiones del Annual Revien of $P$ sychology y las revistas de resúmenes, en especial una relativamente nueva y sobre la que apenas existe información en España: el PsySCAN (Applied Psychology) de la APA.

\section{PRIMER NIVEL: OBRAS EN CASTELLANO}

\subsection{Obras generales:}

BAllesteros, R.: La Psicología aplicada a.la Empresa (2 vol.), Barcelona, CEAC, 1982.

En estos dos volúmenes dedicados el primero a «La empresa y el trabajo. Selección profesional» y el segundo a "La conducta de trabajo. Elementos y condiciones de trabajo", el autor presenta una panorámica bastante completa de la Psicología en el marco de la Empresa. La mejor cualidad del trabajo de Ballesteros es la inmejorable exposición didáctica de los contenidos que se ofrecen. Es la última obra sobre el tema que hasta el presente ha aparecido en castellano y por un autor español.

BluM, M. L., y NAYLOR, J. C.: Psicología Industrial. Sus fundamentos teóricos y sociales. México, Trillas, 1976.

Versión castellana de la $3 .^{a}$ edición inglesa de 1968. (La r. ${ }^{a}$ ed. inglesa data de 1949.) Obra de carácter general que trata de reflejar la etapa de transición por la que pasó la Psicología Industrial en la década de los años 60.

Chiavenato, E.: Introducción a la Teoría General de la Administración. Bogotá, McGraw-Hill Latinoamericana, 198 I.

Presentación y análisis de las distintas teorias sobre Administración.

DESSLER, G.: Organización y Administración. Enfoque situacional, Madrid, Prentice/Hall International, 1979.

Traducción de la edición inglesa de 1976 . Libro sencillo, claro y breve. Recomendable para iniciadores en el tema.

Tiene un especial interés el que sea un libro muy bien documentado y que al final de cada capitulo ofrece un banco de información a través de sus citas que permite profundizar en los distintos temas a través de fuentes directas.

FLEISHMAN, E. A., y BASS, A. R.: Estudios de Psicologia Industrial y del Personal (Eds.). México, Trillas, 1976.

Versión castellana de la edición inglesa de 1961. Es una buena compilación de lecturas que configuran una obra de carácter general y de consulta, resultando especialmente reco- 
mendable por la categoría de los autores de los distintos artículos. Consta de 9 secciones dedicadas a: Desarrollo de criterios de evaluación del desempeño, Selección del personal, Entrenamiento de empleados y gerentes, Motivación, actitudes y satisfacción en el trabajo, Liderazgo y supervisión, La comunicación y el comportamiento en las organizaciones, La fatiga, los accidentes $y$ las condiciones de trabajo, La psicología de ingeniería y Psicología del consumidor.

French, W. L.: Administración de Personal. Desarrollo de recursos bumanos. México, Limusa, 1983.

Versión castellana de la edición inglesa de I 978 , que ha sido revisada y considerablemente ampliada con relación a las anteriores. Dos características del libro son particularmente importantes: Por una parte, el hecho de ofrecer una visión general de los sistemas de organización, $y$, por otra, como su autor dice, supone una amplia aplicación de la teoría contemporánea de la ciencia de la conducta, investigación y práctica de la administración.

Gilmer, B. von H.: Tratado de Psicología Empresarial (2 vols.). Barcelona, Martínez Roca, S. A., 1976.

El primer volumen está dedicado a la Psicología de la Organización y el segundo a las Relaciones Humanas. En conjunto la obra se compone de cinco partes cuyos titulos son:

I. La Psicología de las Organizaciones.

2. Estructura organizativa y dirección empresarial.

3. Motivación y necesidades humanas.

4. Psicología del personal.

9. Hombres y máquinas.

Es versión castellana de la edición inglesa de $197^{1}$.

HAMpton, D. R.; Summer, CH. E., y WeBbER, R. A.: Manual de Desarrollo de Recursos Humanos. México, Trillas, 1982.

Esta edición de Trillas corresponde a la primera edición inglesa de 1973. Se trata de una monumental obra de 845 páginas que en un estilo sencillo y riguroso permite conocer diversos aspectos de las organizaciones como el comportamiento individual, el interpersonal, el grupal y la acción recíproca de los factores humanos y no humanos. El propósito final de los autores eno es cubrir las ciencias del comportamiento, sino descubrir la estructura más honda del comportamiento en las organizaciones y aplicar ese conocimiento pa- ra planificar, administrar y dirigir organizaciones».

Hodgetts, R. M., y Altman, S.: Comportamiento en las Organizaciones. México, Interamericana, 1981 .

Traducción en la ed. inglesa de 1979. Presenta un análisis claro del individuo $y$ la organización, para pasar luego al análisis de grupos, estructuras, proceso y, finalmente, desarrollo organizacional.

HOWELL, W. C.: Psicología Industrial y Organizacional. México, El Manual Moderno, I 979.

Libro introductorio y de carácter general. Aparece bien documentado y, aunque su exposición es muy concisa, resulta claro en su comprensión y fácil en su lectura.

KAST, F. E., y Rosenweig, J. E.: $A d$ ministración en las Organizaciones. Un enfoque de sistemas. México, McGraw-Hill, 1979.

Traducción de la edición inglesa de 1979.

KATZ, D. y KAHN, R. L.: Psicologia Social de las Organizaciones. México, Trillas, 1977.

Versión castellana de la edición inglesa de I966 y que constituye uno de los mejores manuales sobre el tema si bien carece de temas muy importantes y que tuvieron un desarrollo posterior a la creación de la obra. Los autores aprovechan el marco de referencia que ofrece la teoría del sistema abierto para el estudio de las organizaciones a gran escala.

KolB, D. A.; Rubin, I., y MCINTYRE, J. M.: Psicologia de las organizaciones. Problemas contemporáneos. Madrid, Prentice/Hall International (Dossat), 1982.

Tercera reimpresión de la edición castellana de 1977 que, a su vez, es versión de la segunda edición inglesa de 1974. Se trata de una colección de lecturas sobre problemas tales como toma de decisiones en la organización, relaciones grupales, desarrollo de carrera, etc.

\section{Korman, A. K.: Psicologia de la Industria y de las Organizaciones. Madrid, Marova, 1978.}

Traducción del inglés de 1971 . El autor se propone hacer un pequeño tratado de Psicología Industrial como disciplina independiente que vaya más allá de una simple aplicación de 
la Psicología a los problemas industriales y organizativos.

LeVy Leboyer, C.: Psicologia de las Organizaciones. Barcelona, Planeta, 1975.

MAIER, N. R. F.: Psicología Industrial. Madrid, Rialp, I97I.

Texto clásico en lengua castellana. Es una obra de carácter general y muy fácil comprensión. Dado que es una obra excesivamente antigua (versión de la edición inglesa de 1959) su contenido resulta poco válido hoy día. En cualquier caso, no debe faltar en una biblioteca de un estudioso del tema.

MINER, J. B.: Psicología aplicada a la organización bumana de la empresa. Buenos Aires, Paidós, 1977.

Edición inglesa de la que procede la traducción castellana de 1969 .

SCHEIN, E. H.: Psicologia de la Organización. Prentice/Hall International (Dossat). Madrid, 1982.

Existen ediciones anteriores de esta pequeña obra de Schein cuyo primer original inglés es de 1965 . Es una obra recomendable y su contenido se distribuye en cinco partes:

1. El individuo y la organización.

2. Motivación y supuestos sobre la naturaleza humana.

3. Liderazgo y participación.

4. Los grupos en las organizaciones.

3. Estructura y dinámica de la organización.

Smith, C., y Wakeley, H.: Psicologia de la Conducta Industrial. México, McGraw-Hill, 1977.

Es la versión castellana de la tercera edición inglesa. Al igual que las anteriores, se puede considerar como una introducción a la materia, ofreciendo una panorámica general correctamente documentada, pero no llega a profundizar en ningún tema. Son libros de muy fácil lectura.

Strauss, G., y Sayles, L. R.: Personal. Problemas bumanos de la Administración. Madrid, Prentice/Hall International (Dossat), 1981 .

Ediciones inglesas en 1960, 1966, 1967, 1972 y 1980. El libro consta de un total de ocho partes:

I. Los individuos y sus oficios.

2. Grupos, supervisores y sindicatos.
3. Destrezas gerenciales.

4. La estructura.

5. Desarrollo y protección del trabajador.

6. La Administración y el desarrolloorganizacional.

7. La remuneración.

8. Conclusión.

WERTHER, W. B. Jr., y DAVIS, K.: Dirección de Personal y Recursos Humanos. México, McGraw-Hill, 1982.

Edición inglesa de 1981. Libro muy actualizado. Parece particularmente recomendable por las temáticas que trata. A las habituales en este tipo de tratados añade:

- Problemas ambientales.

- Calidad de vida de trabajo.

- Relaciones obrero-patronales.

- Auditoria de personal, etc.

\subsection{Obras especificas}

ARGYRIS, CH.: El individuo dentro de la organización. Barcelona, Herder, I 979.

Edición inglesa de 1964. Conforme reza el título de la obra, se trata, fundamentalmente, de considerar la potencialidad del individuo dentro de la organización y desarrollarla al máximo en bien del individuo y de la organización. Tras ofrecer diversos análisis, el autor ofrece diversas vías a través de las cuales se podrían evitar o reducir las consecuencias no deseadas que inciden en el individuo y en la organización.

BARLETT, A. C., y KAYSER, T. A.: Cambio de la Conducta Organizacional. México, Trillas, 1980.

Traducción de la compilación inglesa editada en 1973. Trata de ofrecer una visión equilibrada del proceso de cambio organizacional sin detenerse excesivamente en unos aspectos y no en otros. Al lado de aspectos relacionados con el desarrollo organizacional y el entretenimiento de laboratorio, se contemplan otros relacionados con aspectos estructurales o técnicos.

Blake, R. R., y MOUton, J. S.: El Nuevo Grid Gerencial. México, Diana, I980.

Chevalier, A.: El balance social de la empresa. Madrid, Fundación Universidad Empresa, 1977.

Dowling, W. F. Jr., y SAYles, L. R.: 
Mando y motivación efectiva del personal. Barcelona, Hispano Europea, 1979.

No pone la fecha de la edición inglesa de la que procede la castellana.

DRUCKER, P. F.: La Gerencia de Empresas. Barcelona, EDHASA, 1979.

Traducción de la edición inglesa de 1954. Es un autor clásico en este tema.

DUNnETTE, M.: Selección y Administración de Personal. México, CECSA, 1980.

Primera edición, de 1970.

Fertinanti, M., y Grosso, C. A.: Análisisy Valoración de Tareas. Bilbao, Deusto, 1978 .

FORRESTER, J. W.: Dinámica Industrial. El Ateneo. Buenos Aires, 1972.

FRASER, J. M.: La entrevista de Selección de Personal. Deusto. Bilbao, 1983.

Giegold, W. C.: APO (Serie de cuatro volúmenes sobre Evaluación de Rendimiento. Planificación estratégica, Establecimiento de objetivos y $\mathrm{El}$ proceso de Administración por objetivos, junto con el Manual del instructor). México, McGraw-Hill, 1982.

Versión castellana de la ed. inglesa de $197^{8}$.

KOLD, D. A.; RUBIN, I., y MCINTYRE, J. M.: Psicologia de las organizaciones. Experiencias. Prentice/Hall International (Dossat). Madrid, 1982.

Cuarta reimpresión de la versión castellana de 1976, traducción de la segunda edición inglesa de 1974. Trata de mostrar ciertos principios generales de Psicología y su aplicabilidad en sociedades y empresas, estableciendo un interesante vinculo de enlace entre el carácter general de toda ciencia y su materialización en la realidad concreta.

Morgan, H., y Cogger, J.: Manual del entrevistador. TEA. Madrid, 1977.

OFICINA INTERNACIONAL DEL TRABA. JO: Clasificación Internacional Uniforme de Ocupaciones (CIUO). OIT. Ginebra, 1968. Edición revisada de la de 1958.

Ordena de forma sistemática las ocupaciones del total de la población civil activa. Su estructura presenta cuatro niveles, cada uno de los cuales ofrece una exposición más deta- llada que el inmediato anterior: ocho grandes grupos, 83 subgrupos, 284 grupos primarios y 1.506 categorías ocupacionales. Cada una de las 1.881 denominaciones va acompañada de su correspondiente defínición.

OFICINA INTERNACIONAL DEL TRABAJO: Introducción al Estudio del Trabajo. OIT. Ginebra, 1980.

Tercera edición revisada. Consta de cuatro partes: Productividad y estudio del trabajo, Estudio de métodos, Medición del trabajo y Nuevas formas de organización del trabajo.

OUCHI, W.: Teoría Z. Cómo pueden las empresas bacer frente al desafio japonés. Fondo Educativo Interamericano. México, 1982.

Edición inglesa de $198 \mathrm{I}$.

SAYLES, L. R.: Liderazgo. Estilos y Técnicas. McGraw-Hill. México, 1982.

Edición inglesa de 1979.

SCHEIN, E. H., y BENNIS, W. G.: El cambio personal y organizacional a través de métodos grupales. Herder. Barcelona, 1980.

El original inglés es de 1975. Obra colectiva en la que han participado un total de 17 autores y que trata de mostrar el valor potencial de la formación de laboratorio y en particular de los "Training Groups" y su aplicabilidad a nivel individual y organizacional.

Tregoe, B. B., y Zimmerman, J. W.: Estrategia de Alta Dirección. Su Naturaleza y Aplicación. Interamericana. México, 1983.

De la edición inglesa de 1980 .

VROOM, V. H., y DECI, E. L. (Eds.): Motivación y Alta Dirección. Trillas. México, 1979.

Del inglés de 1970. Colección de lecturas de grandes autores cläsicos.

\subsection{Colecciones}

Existen numerosas editoriales que tienen colecciones específicas relacionadas con la conducta en la organización laboral. Sin pretender ser exhaustivos vamos a mencionar algunas de las más destacadas. 
La Editorial Interamericana inició en 1982 una "Biblioteca de Desarrollo Gerencial» con obras de carácter muy elemental y accesibles a un público no especializado. Hasta la fecha esta colección cuenta con títulos como:

BAEHLER: Guia del Exito Gerencial.

BROADWELL: La Guia del Supervisor Actual.

BELKER: De Empleado a Gerente. Guia práctica de Dirección de personal.

COOPER: Comunicación no verbal para ejecutivos.

KOTTER: El poder gerencial. Cómo reconocerlo, obtenerlo y usarlo.

PINkSTafF y PINKSTAFF: Cómo desarrollar sus babilidades gerenciales.

DOWLING y DROLET: Cómo desarrollar un programa de capacitación y entrenamiento.

El Fondo Educativo Interamericano, a través de su colección de «Desarrollo Organizacional» trata de presentar teorías y estrategias de cambio que orienten a la organización hacia la óptima utilización de sus recursos. Algunas obras de la serie son:

BENNIS: Desarrollo organizacional: Su naturaleza, sus origenes y perspectivas.

BECKHARD: Desarrollo organizacional: Estrategias y modelos.

BLAKE y MOUTON: O. $c$.

LAWRENCE/LORSCH: Desarrollo de organizaciones: Diagnóstico y acción.

WALTON: Conciliación de conflictos interpersonales: confrontaciones y consultoria de mediadores.

SCHFIN: Consultoria de procesos: Su papel en el desarrollo organizacional.

FORDYCE/WFIL: Métodos de desarrollo organizacional para ejecutivos.

GALBRAITH: Planificación de organizaciones.

PARTIN: Perspectivas del Desarrollo Organizacional.

La Editorial Hispano Europea tiene también abundante material de calidad contrastada en el campo de la organización industrial y de la administración de empresas, destacando su «Colección Esade» con títulos como:
HACON, R.: Eficiencia personal y de la organización.

PEÑA BATZAN, M.: Dirección de personal.

MCGREGOR, D.: La participación en la industria.

VITELES, M. A.: Motivación y Moral en la Industria.

Ediciones Deusto también tiene abundante material de psicologia industrial, pero de un interés muy desigual, predominando claramente lo divulgativo. Destaca sobre todo su serie «Harvard Deusto", selección de artículos traducidos del "Harvard Business Review".

En Ediciones Rialp también se encuentra mucha bibliografía del tema, predominando las obras de corte clásico.

Ibérico Europea de Ediciones en su colección «La Empresa Moderna» tiene diez series de temas empresariales en una línea editorial claramente divulgativa.

Aun quedándonos numerosas colecciones en el tintero, para hacer un poco de justicia queremos destacar también la labor editorial de las editoriales mejicanas Trillas, con su colección «Biblioteca de Ciencias de la Administración” y El Manual Moderno, cuyos títulos destacan siempre por su calidad. CECSA, con su serie de "Sociología Industrial", de carácter más divulgativo. Finalmente, señalar que en el Catálogo de Publicaciones de la APD existen también algunas publicaciones de interés.

\section{SEGUNDO NIVEL}

\section{I. Obras generales o integradoras}

BedeIAN, A. G.: Organizations: Theory and Analysis. Hinsdale. Dryden Press. Illinois, I980 ${ }^{1}$.

DubIN, R. (ed.): Handbook of Work. Organization and Society. Rand McNally. Chicago, 1976.

DUNNETTE, M. E. (ed.): Handbook of Industrial and Organizational Psychology. Rand McNally. Chicago, 1976. 
Es la mejor obra realizada hasta el momento en el campo de la Psicologia Industrial y Organizacional. El número de temas tratados, la profundidad de su tratamiento y la misma calidad de los autores coordinados por Dunnette, hacen que ésta sea una obra verdaderamente importante.

Landy, F. J., y Trumbo, D. A.: Psychology of Work Behavior. Homewood. Dorsey Press. Illinois, $1980^{2}$.

MCCORMICK, E. J., y ILGEN, D. R.: Industrial Psychology. Prentice-Hall. Nueva York, 1980 ( $7 . .^{2}$ ed.) ${ }^{3}$.

MCCORMICK, E. J., y SANDERS, M. S.: Human Factors in Engineering and Design. McGraw-Hill. Nueva York, 1982 (.$^{\mathrm{a}}$ ed. $)^{4}$.

MILES, R. H.: Macro Organizational Bebavior: The Goodyear series in Administration and Bussiness Management. Goodyear. Santa Mónica. California, 1980 5.

MINER, J. B.: Theories of Organizational Behavior. Dryden. Hinsdale, Illinois, 1980.

Mitchell, T. R.: People in Organization: An Introduction to Organizational Bebavior. McGraw-Hill. Nueva York, 1982 (2. ${ }^{2}$ ed. $)^{6}$.

MOHR, L. B.: Explaining Organizational Behavior. Jossey-Bass. San Francisco, 1982 ?.

NAYloR, J. C.; PRITChaRd, R. D., e ILGEN, D. R.: $A$ Theory of Bebavior in Organizations. Academic Press. Nueva York, $1980^{8}$.

NYSTROM, P. C., y STARBUCK, W. H. (Eds.): Handbook of Organizational Design. Oxford University Press. Nueva York, 1981 (2 vols.).

PORTER, L. W.; LAWLER, E. E. III, y HACKMAN, J. R.: Bebavior in Organizations. McGraw-Hill. Nueva York, i 975.

SCHERMERHORN, J. R. Jr.; HUNT, J. G., y OSBORN, R. M.: Managing Organizational Bebavior. Wiley. Nueva York, 1982 ?.

SImmONS, R. H.: Achieving Human Organization. Spencer. Malibu. California, $19^{81}{ }^{10}$.

STEERS, R. M.: Introduction to Organizational Behavior. Goodyear. Santa Mónica. California, 1981.

WEXLEY, K. N., y YUKL, G. A.: Orga- nizational Behavior and Industrial Psychology. Readings with commentary. Oxford University Press. Oxford, 197?.

\subsection{Textos especializados o monografias}

AMERICAN PSYCHOLOGICAL ASSOCIATION DIVISION OF INDUSTRIAL-ORGANIZATIONAL PSYCHOLOGY: Principles for the Validation and Use of Personnel Selection Procedures. Div. Ind. Organiz. Psych. Berkeley. California, 1980. 2. ${ }^{2}$ edición.

BowDITCH, J. L., y BUONO, A. F.: Quality of Work Life Assessment. A Survey-Based Approaich. Auburn House Publishing Co. Boston, 1982.

CASCIO, W. F., y AWAD, E. M.: Human Resources Management: An Information Systems Approach. Reston. Reston. Va., 198111.

GolembIEWSKI, R. T.: Approaches to Planned Change. Dekker. Nueva York, 1979. Dos volúmenes: I) Orienting Perspectives and Micro-Level Interventions; 2) Macro-Level Interventions and Change-Agent Strategies 12.

GRUNEBERG, M. M.: Understanding Job Satisfaction. Halsted Press. Nueva York, $1979^{13}$.

Kimberly, J. R.; Miles, R. H., et al.: The Organizational Life Cycle: Issues in the Creation, Transformation and Decline of Organization. Jossey-Bass. San Francisco, $1980^{14}$.

LAWLER, E. E. III.: Motivation in Work Organizations. Brooks/Cole. Monterrey, California, 1973.

LAWLER, E. E. III.: Pay and Organization Development. Addison-Wesley. Reading. Mass., 198 I 15.

LEVINSON, H.; MOLINARI, J., y SPOHN, A. G.: Organizational Diagnosis. Harvard Univ. Press. Cambridge, Mass., 1972.

MOBLEY, W. H.: Empleyee Turnover: Causes, Consequences and Control. AddisonWesley. Reading. Mass., $1982{ }^{16}$.

Pfeffer, J.: Power in Organizations. Pitman. Marshfield, Mass., 198 I ${ }^{17}$.

Ross, J. E.: Productivity, People and Profits. Reston. Reston, Va., $1981{ }^{18}$. 
Sternthal, B., y CRAIG, C. S.: Consumer Bebavior: An Information Processing Perspective. Prentice/Hall International. Nueva York, $1982{ }^{19}$.

TANNENBAUM, R.; WESCHLER, I. R., y MASSARIK, F.: Leadership and Organization. A Behavioral Science Perspective. McGraw-Hill. Nueva York, 1961 .

UNGERSON, B.: Recruitment Handbook. Gower. Aldershot, Hants, 1983. ( r $^{\text {a }}$ edición 1970).

VROON, V. H.: Work and Motivation. Wiley. Nueva York, 1964.

\subsection{Compilaciones}

Alluisi, E. A., y Fleishman, E. (Eds.): Human Perfomance and Productivity: Stress and Effectiveness. Lawrence Erlbaum Ass. Hillsdale. New Jersey, 1982 (vol. núm. 3 de una serie de 3 ).

COOPER, C. L., y PAYNE, R. (Eds.): Current Concerns in Occupational Stress. Wiley. Chichester. England, I980 20.

CORLETT, E. N., y RICHARDSON, J. (Eds.): Stress. Work Design and Productivity. Wiley. Chichester. England, $1982{ }^{21}$.

Cummings, T. G. (Ed.): Systems theory for Organization Development. Wiley. Chichester. England, I 98022.

Cummings, L. L., y STAw, B. M. (Eds.): Research in Organizational Bebavior: $A n$ Annual Series of Analytical Essays and Critical Reviews. Vol. 3. JAI Press. Greenwich, Conn., 1981 .

Dunnette, M. D., y Fleishman, E. A. (Eds.): Human Perfomance and Productivity: Human Capability Assessment. Lawrence Erlbaum Ass., Hillsdale, New Jersey, I982. (Vol. núm. I de una serie de 3).

EGDAHL, R. H., y CHAPMAN, W. D. (Eds.): Mental Wellness Programs for Employees. Springer-Verlag. Nueva York, 1980.

Este volumen forma parte de una serie que bajo el título Industry and Health Care edita Springer-Verlag. En 1980 se habian publicado ya ocho volúmenes. El de Egdahl y Chapman es el número 9 .
ENGLAND, G. W.; NEGANDHI, A. R., y WILPERT, B. Eds):: The Functioning of Complex Organizations. Oelgeschlager, Gunn and Hain. Cambridge, Mass., 198123 .

EvaN, W. M.: Frontiers in Organization and Management. Praeger. Nueva York, 198024.

HERBERT, Th. T. (Ed.): Organizational Behavior. Readings and Cases. MacMillan. Nueva York, 1976.

HOLDING, D. H. (Ed.): Experimental Psycbology in Industry. Penguin Books. Harmondsworth, Middlesex, 1969.

Howell, W. C., y Fleishman, E. A. (Eds.): Human Performance and Productivity: Information and Decision Making. (Vol. núm. 2 de una serie de 3). Lawrence Erlbaum Ass. Hillsdale, New Jersey, 1982 .

HunT, J. G., SERAKaN, U., y Schries. HEIM, CH. A. (Eds.): Leadership: Beyond Establishment Views. Southern Illinois University. Carbondale, 198225.

Este es el 6.० volumen de «Leadership Symposia Series" que tienen lugar periódicamente desde 1973.

KATZ, D.; KAHN, R. L., y ADAMS, J. S. (Eds.): The Study of Organizations. Jossey-Bass. San Francisco, 198026.

LAWLER, E. E. III; NADleR, D. A., y CAMMANN, C. (Eds.): Organizational Assessment: Perspectives on the Measurement of Organizational Behavior and the Quality of Work Life. Wiley Interscience. Nueva York, 1980.

MORAY, N. (Ed.): Mental Workload. Its Theory and Measurement. Plenum Press. Nueva York, I979.

Este volumen forma parte de la tercera serie de Conferencias de la NATO dedicada a "Human Factors». Esta serie constaba ya en 1981 de 17 volúmenes de los que nosotros destacamos los siguientes:

Volumen núm. I: «Monitoring Behavior and Supervisory Control» (Ed. por Th. B. Sheridan y G. Johannsen).

Volumen núm. 6: «Language Interpretation and Communication" (Ed. por D. Gerver y W. Sinaiko). 
Volumen núm. II: "The Analysis of Social Skill» (Ed. por W. T. Singleton, P. Spurgeon y R. B. Stammers).

Volumen núm. 17: «Manned Systems Desing: Methods, Equipment and Application» (Ed. por Moraal y K. F. Kraiss).

NORD, W. (Ed.): Concepts and Controversy in Organizational Bebavior. Goodyear Publishing Company. Pacific Palisades, Calif., I 972.

Staw, B. M., y Cummings, L. L. (Eds.): Research in Organizational Behavior: An Annual Series of Analytical Essays and Critical Reviews. Vol. 4, 1982, JAI Press, Greewich, Coinn. 198228.

STAW, B. M., y SALANCIK, G. R. (Eds.): New Directions in Organizational Behavior. St. Clair. Chicago, 1977.

STEPHENSON, G. M., y BROTHERTON, CH. (Eds.): Industrial Relations. A Social Psychological Approach. John Wiley and Sons. New York, 1979.

\section{ร. TERCER NIVEL}

Como se indicó anteriormente, el tercer nivel contiene una fuente primaria - las revistas - y dos fuentes secundarias que son las revisiones y las revistas de resúmenes. Como se sabe, fuentes primarias son aquellas que proporcionan la información directamente y fuentes secundarias las que remiten a otras, facilitando su localización.

En este apartado las revistas seleccionadas aparecerán en tercer lugar, ya que para su selección se ha utilizado la intersección de dos conjuntos: las revistas que, a juicio de los autores, aparecian cuantitativa y cualitativamente más representadas en las revisiones del Annual Review y las revistas preferidas por los usuarios del Psycscan en lo concerniente a la psicología organizacional.

\section{S.I. Revisiones}

El Annual Review of Psychology es una publicación que, desde hace más de treinta años, publica exámenes de la literatura científica de diversas áreas de la Psicolo- gia. Una de las temáticas incluida tradicionalmente en los esquemas organizativos de esta fuente documental ha sido la Psicología Organizacional e Industrial. El estudioso puede seguir la evolución conceptual de este campo siguiendo las revisiones que, desde el mismo volumen $\mathbf{1}$, en 1950 , comienzan a publicarse primero bajo el epígrafe Industrial Psychology y, posteriormente, con títulos como. Organizational Psychology.

A continuación, se presenta una lista de las diversas revisiones que sobre Psicología Organizacional y temas afines han aparecido en el Annual Review. Para facilitar su consulta se ha realizado una organización elemental por materias.

\subsubsection{Industrial Psychology (Psicologia industrial)}

Shartle, C. L.: «Industrial Psychology». A.R.P. I950, I, I I I-172.

BELlows, R. M.: «Industrial Psychology». A.R.P. 1951, 2, 73-192.

BROWN, C. B.; GHISELLI, E. E.: «Industrial Psychology». A.R.P. I952, 3, 205232.

Harrell, T. W.: «Industrial Psychology». A.R.P. 1953, 4, 21 5-238.

HERON, A.: «Industrial Psychology». A.R.P. 1954, 5, 203-228.

WALLACE, Jr., S. R.; WEITZ, J.: «Industrial Psychology". A.R.P. 1956, 7, 197232.

KATZELl, R. A.: «Industrial Psychology». A.R.P. 1957, 8, 237-268.

FERGUSON, L. W.: «Industrial Psychology». A.R.P. 1958, 9, 243-266.

GILMER, B. VON H.: «Industrial Psychology». A.R.P. 1960, 11, 323-350.

VROOM, V. H.; MAIER, N. R. F.: «Industrial Social Psychology". A.R.P. I 961, I2, 41 3-446.

\subsubsection{Personnel Management}

(Dirección de personal)

DunNeTTE, M. D.: «Personnel Management». A.R.P. 1962, 13, 28 5-314. 
SELLS, S. B.: «Personnel Management». A.R.P. 1964, Is, 399-420.

PORTER, L. W.: «Personnel Management». A.R.P. 1966, 17, 395-422.

\subsubsection{Psychology of Men at Work} (Psicologia del trabajo)

SMITH, P. C.; CRANNY, C. J.: «Psychology of Men at Work». A.R.P. 1968, $19,467-496$.

HINRICHS, J. R.: «Psychology of Men at Work». A.R.P. 1970, 21 , 519-5 54.

\subsubsection{Organizational Psychology}

(Psicología de las organizaciones)

LEAVITT, H. J.; BASS, B. M.: «Organizational Psychology». A.R.P. I964, Is, 37 I-398.

QUINN, R. P.; KAHN, R. L.: «Organizational Psychology». A.R.P. 1967, 18, 437-446.

MITCHELL, T. R.: «Organizational Behavior». A.R.P. 1979, 30, 243-28 I.

CUMMINGS, L. L.: "Organizational Behavior"). A.R.P. 1982, 33, 541-579.

\subsubsection{Organizational Development} (Desarrollo organizacional)

FRIEDLANDER, F.; BROWN, L. D.: «Organizational Development». A.R.P. 1974, 25, 313-341.

ALDERFER, C. P.: «Organizational Development». A.R.P. 1977, 28, 197-223.

FaucheuX, C.; AMADO, G., y LAU. RENT, A.: "Organizational Development and Change». A.R.P. 198 2, 33 , 343-370.

\subsubsection{Personnel Selection} (Selección de personal)

TAYloR, E. K.; NeVIS, E. C.: «Personnel Selection». A.R.P. 1961, 12, 3894 I 2.
DUDEK, E. E.: «Personnel Selection». A.R.P. I 963, I4, 261-284.

BIESHEUVEL, S.: «Personnel Selection». A.R.P. $1965,16,295-324$.

GuION, R. M.: «Personnel Selection». A.R.P. 1967, 18, 191-216.

OWENS, W. A.; JEWELL, D. O.: «Personnel Selection». A.R.P. 1969, 20, 419446.

BRAY, D. W.; MOSES, J. L.: «Personnel Selection». A.R.P. 1972, 23, 545-576.

ASH, PH.; KROEKER, L. P.: «Personnel Selection». Classification and Placement. A.R.P. 1975, 26, 481-507.

DUNnETTE, M. D.; BORMAN, W. C.: «Personnel Selecion». Classification. Systems. A.R.P. 1979, 30, 477-529.

TENDPYR, M. L.; DELTJEN, P. D.: «Personnel Selecion». Classifacation. A.R.P. 1982, 33, 581-618.

\subsubsection{Consumer Psychology \\ (Psicología del consumidor)}

GUEST, L.: «Consumer Analysis». A.R.P. $1962,13,319-344$.

TWEDT, D. W.: "Consumer Analysis". A.R.P. 1965, 16, $265-294$.

PERLOFF, R.: "Consumer Analysis». A.R.P. 1968, 19, 437-466.

JACOBY, J.: "Consumer Psychology: An Octenium». A.R.P. 1976, 27, 33 1-358.

KASSARJIAN, H. H.: "Consumer Psychology». A.R.P. 1982, 33, 619-649.

\subsubsection{Engineering Psycbology (Ergonomía)}

FITTS, P. M.: «Engineering Psychology». A.R.P. 1958, 9, 267-294.

MELTON, A. W.; BRIGGS, G. E.: «Engineering Psychology». A.R.P. 1960, II, $71-98$.

CHAPANIS, A.: "Engineering Psychology». A.R.P. 1963, 14, $285-318$.

POULTON, E. C.: "Engineering Psychology». A.R.P. 1966, 17, 1 77-200.

Alluisi, E. A.; MORGAN, B. B. (Jr.): «Engineering Psychology. Human and Performance». A.R.P. 1976, 27, 305330. 
S.1.9. Personnel Attitudes and Motivation (Actitudes y motivación del personal)

Miliver, J. B.; DACHLER, H. P.: «Personnel Attitudes and Motivation». A.R.P. 1973, 24, 379-402.

LOCKE, E. A.: "Personnel Attitudes and Motivation». A.R.P. 1975, 26, 45 7-480.

Korman, A. K.; Greenhaus, J.; BADIN, I. J.: «Personnel Attitudes and Motivation». A.R.P. 1977, 28, 175-196.

5.1.10. Personnel Training and Development (Desarrollo y aprendizaje del personal)

CAMPBEll, J.: «Personnel Training and Development». A.R.P. 1971, 22, 565602.

HELler, F. A.; ClaRK, A. W.: «Personnel and Human Resources Development». A.R.P. 1 976, 27, 405-435.

GOLDSTEIN, I. L.: "Training in Work Organizations". A.R.P. 1980, 31, 229272.

\subsection{Revistas de resúmenes}

Las revistas de resúmenes son publicaciones que recogen, ordenadas temáticamente, la descripción bibliográfica de artículos y otras publicaciones junto con un resumen acerca de su contenido.

5.2.I. La revista de resúmenes más popular en Psicología es el Psychological Abstracts; el lector interesado puede consultar su estructura y utilización en Fernández Dols y Ortega (1980). En el Psychological Abstracts existen numerosos descriptores para clasificar publicaciones relacionadas con la Psicología Organizacional. Por ejemplo: Industrial Psycbology (en lingar de Organizational Psychology), Business Management, Business Organizations, Occupations (en lugar de Careers, Jobs y Vocations), Organizational Behavior, Organizational Change, Organizational Development, Working Conditions, etcétera.

9.2.2. Existen, además, otras revistas de resúmenes relacionadas genéricamente con el mundo del trabajo; describiremos las más importantes siguiendo a McInnis (1982) y el Ulrich's International Periodicals Directory (1981).

El Boletín Bibliográfico del Servicio Social de Higiene y Seguridad del Trabajo recoge resúmenes de publicaciones que tratan aspectos relativos a la higiene y el medio ambiente del trabajo en general; a la Fisiología del Trabajo y Ergonomía; consecuencias biológicas de las exposiciones peligrosas; enfermedades profesionales; seguridad; medicina de la empresa; legislación, Psicología, Sociología y Pedagogía encaminadas a la prevención de riesgos profesionales. Su periodicidad es mensual y posee un índice temático acumulativo anual.

El Ergonomics Abstracts recoge unos diez mil resúmenes de diversos tipos de documentos ordenados por categorías gracias a un completo tesauro. Se comenzó a publicar en 1965.

Human Resources Abstracts (antes: Poverty and Human Resources Abstracts) recoge resúmenes sobre documentos de habla inglesa en torno a problemas del mercado de trabajo, fuerza de trabajo, empleo y formación profesional, etc. Se publica desde 1966.

El International Labour Documentation recoge información sobre todo tipo de documentos relacionados con el mundo del trabajo. Su organización se basa en el tesauro de la OIT y posee índices alfabéticos en inglés, francés y español de los descriptores para los documentos indizados. Se publica desde 1965 y su periodicidad es mensual.

Personnel Management Abstracts se publica trimestralmente desde 1955; recoge información sobre el mundo de la dirección de personal, tanto desde un punto de vista académico como profesional.

Work Related Abstracts recopila información sobre artículos procedentes de más de 250 publicaciones en torno a 20 temáticas, tales como conducta humana y trabajo, dirección de personal, higiene y 
seguridad, etc. Se publica mensualmente desde 1950, posee índices acumulativos trimestrales y anuales cuya organización temática corresponde a un elaborado tesauro que se revisa anualmente.

5.2.3. Además de las revistas de resúmenes, que o bien se centran en el mundo de la Psicología en general o bien en el mundo del trabajo, existe una publicación, de aparición reciente, que quizá sea la más ajustada a las necesidades del psicólogo, al menos en lo que se refiere a la información del área de influencia anglosajona.

Se trata del Psycscan: Applied Psychology, publicación de periodicidad trimestral que pertenece a una serie de publicaciones editadas por la APA que poseen una estructura similar, aunque no idéntica, al Psychological Abstracts.

El Psycscan: Applied Psychology es considerablemente más barato que el Psychological Abstracts y cubre el área especifica de la Psicología Aplicada, desglosada, por ejemplo (vol. I, núm. 2), en los siguientes apartados: General; Seguridad y accidentes; Consumo y marketing; Ambiental; Psicología del Derecho; Ergonomia; Organizaciones; Psicología Ocupacional; Psicometría Aplicada; Psicología Social Aplicada y Psicología del Deporte.

La filosofia del Psycscan consiste en resumir (en unos 400 abstracts al trimestre) la información más relevante aparecida sobre un área específica en el Psycbological Abstracts y proporcionársela al profesional de forma más barata y accesible. Para ello se ha modificado ligeramente la presentación del abstract o resumen y, lo que es más importante, son los propios suscriptores los que deciden periódicamente qué revistas son las que debe cubrir Psyscan hasta, aproximadamente, medio centenar de publicaciones. Por último, el precio de suscripción anual para el Psycsan es de unas 2.000 pesetas, frente a las, aproximadamente, 75.000 pesetas al año que supone la sucripción al Psychological Abstracts (Psycscan. American Psychological Association I 200 I 7 th street N. W. Washington D. C. 20036).

\subsection{Revistas}

5.3.1. Existen en castellano diversas publicaciones que, en algunos casos, pueden tener interés para el psicólogo encuadrado profesionalmente en una organización. Es el caso, entre otras, de la Revista de Politica social (editada por el Centro de Estudios Constitucionales, plaza de la Marina Española, Madrid-14); Aedipe (boletín de la Asociación Española de Dirección de Personal, c/ Moreto, 10, Madrid14); Alta Dirección (Ed. Nauta, Loreto, 16, Barcelona-29) y la Revista del Trabajo publicada por el Ministerio de Trabajo (Servicio de Publicaciones, plaza San Juan de la Cruz, Nuevos Ministerios, Madrid).

5.3.2. La información específica sobre los avances técnicos en el campo específico de la Psicología proviene, sin embargo, del área anglosajona.

Seria imposible recoger aquí todas las revistas importantes, pero puede ser un muestreo representativo el que consiste en seleccionar aquellas revistas cubiertas por el Psycscan que también parecen ser cuantitativa y cualitativamente importantes en las revisiones del Annual Revien of Psychology (vid. supra).

A continuación, se presentan los once títulos que resultan con una breve descripción, procedente de la XX edición del Ulrich's International Periodical Directory (198I), que incluye datos de gran interés práctico.

I. Academy of Management Journal (inicio en 1958), la publica trimestralmente la Academy of Mangement (suscripciones: F. Ray Box K2, Mississippi State, MS 39762).

2. Administrative Science Quarterly (inicio en 1956) (dirección: Cornell Univ., Graduate School of Bussiness and Public Administration, Ithace, N. Y., 14853 .

3. Human Factors Society Bulletin (desde 1958), mensual (Human Factors Society, Box 1369 Santa Monica, CA 90406). 


\section{Estudios}

4. Human Relations (1947), mensual; representa la línea del Tavistock Instituye of Human Relations, pero las suscripciones corren a cargo de Plenum Publishing Co.

5. Journal of Applied Psychology (1917), bimensual. Publicada por la APA (vid. supra).

6. Journal of Consumer Research (desde 1974), trimestral; lo edita, en el sentido intelectual, el University of Illinois and Chicago Circle (para suscripciones: $222 \mathrm{~S}$. Riverside, Chicago, Ill., 60606).

7. Journal of Vocational Bebavior (desde 1971), aparece seis veces al año y su edición corre a cargo de una importante compañia editorial (Academic Press).

8. Organizational Behavior and Human
Performance ( $« \mathrm{~A}$ journal of fundamental research and theory in Applied Psychology»). Es bimensual y, como la anterior, pertenece a Academic Press.

9. Personnel and Guidance Journal (desde 1922), es mensual, excepto en los meses de verano. La edita la American Personnel and Guidance Association (Two Skyline Pl., ste. 400, 52003 Leersburg Pike, Falls Church, VA 2204I).

10. Personnel Psychology, se trata de una publicación trimestral (suscripciones: Box 6956, College Sta., Durkam, NC 27708).

I1. Public Personnel Management, publicada trimestralmente desde 1976 por la Academy of Management (vid. supra la revista del mismo nombre).

\section{Referencias}

DunnetTe, M. D.: "Toward Fusion" en Dunnete, M. D. (ed.): Handbook of Industrial and Organizational Psycbology. Chicago, Roand McNally, 1976.

FernÁndez Dols, J. M., y OrTega, J. E.: Fuentes documentales en Psicologia. Madrid, Debate, 1980.

PuGH, D. S., et al.: Writers on Organizations (2nd. ed.). Harmondsworth, Penguin, 1971.

McINNis, R. G.: Research Guide for Psycbology. Westport, Conn., Greenwood, 1982.

Ulirich's International Periodical Directory: 20 th ed., Nueva York, Bowker, 1981.

\section{Notas}

1 Véase Contemporary Psychology, 26, 8, pág. 646, 1981.

Véase Contemporary Psycbology, 27, 1, págs. 31-32, $198 \mathrm{I}$.

Véase Contemporary Psychology, 26, 8, págs. 617-618, 1981.

Véase Contemporary Psychology, 28, 1, págs. 57-58, 1983.

Véase Contemporary Psycbology, 27, 1, pág. 59, 1981.

Véase Contemporary Psychology, 23, 3, pág. 218, 1983.

Véase Contemporary Psychology, 28, 5, págs. $350-351, x 983$.

Véase Contemporary Psychology, 26, 9, págs. 661-663, 1981 .

Véase Contemporary Psycbology, 28, 3, págs. $225-226,1983$.

Véase Contemporary Psycbology, 28, 3, págs. 221 1-222, 1983.

Véase Contemporary Psycbology, 27, 8, págs. 629-630, 1982.

Véase Contemporary Psychology, 26, 3, págs. 201-203, 1981.

Véase Contemporary Psychology, 26, 5, págs. $381-382,1981$.

14 Véase Contemporary Psychology, 26, 10, págs. 776-777, 1981 .

Véase Contemporary Psychology, 27, 4, págs. 282-284, 1982.

16 Véase Contemporary Psychology, 28, 3, págs. 223-224, 1983.

Véase Contemporary Psycbology, 27, 1, págs. 42-43, 1982.

18 Véase Contemporary Psychology, 27, 9, pág. 732, 1982.

19 Véase Contemporary Psychology, 28, 4, págs. 307-308, 1983.

20 Véase Contemporary Pychology, 26, 4, pág. 300, 1981.

21 Véase Contemporary Psychology, 28, 4, págs. 309-310, 1983. 
22 Véase Contemporary Psychology, 27, 1, págs. 37-38, 1982.

23 Véase Contemporary Psycbology, 27, 9, págs. 707-708, 1982.

24 Véase Contemporary Psychology, 26, 5, pág. 358, 1981.

25 Véase Contemporary Psycbology, 28, 3, págs. $214^{-216}, 1983$.

26 Véase Contemporary Psycbology, 27, 1, págs. 26-27, 1982.

27 Véase Contemporary Psychology, 26, 7, págs. 546-547, $198 \mathrm{I}$.

28 Véase Contemporary Psycbology, 28, 5, págs. 404-405, 1983.

29 Véase Rodriguez Sanabria, F., y Fernandez Dols, J. M.: Estudios de Psicologia, núm. 9, páginas $143^{-1} 57,1982$.

30 Véase Jiménez, A.; González, M.; Muñoz, C.; Mallo, M. J., y Fernández Dols, J. M.: Estudios de Psicologia, núm. 13, págs. $1111-121,19^{8} 3$.

31 Véase Jiménez, A.; Carrobles, J. A. I.; Gomez, C.; Calzada, M. J.; Muñoz, P., y Fernández Dols, J. M.: Estudios de Psicologia, núms. 14-15, págs. 152-165, 1983.

32 Véase Delci.aux, I.; Fernández Dols, J. M.; Jiménez, A.: Guia de Computadores y Psicología (en prensa). 